\title{
Letters
}

\section{Comments on "Internal Impedance of Conductors of Rectangular Cross Section"}

\author{
F. Medina and R. Marqués
}

In the above paper, ${ }^{1}$ Antonini et al. discuss some interesting questions concerning the ac internal impedance of rectangular cross-sectional conductors (such as those typically used as transmission lines and interconnects in many microwave and digital printed circuits). The authors of the above paper present calculations showing that the real $(R)$ and the imaginary $\left(\omega L_{i}\right)$ parts of the alternate current (ac) internal impedance $\left(Z=R+j \omega L_{i}\right)$ are not identical for that type of conductors even if a strong skin-effect condition prevails. Following the authors discussion, this would be in sharp contrast with the equality $\left(R=\omega L_{i}\right)$ commonly accepted in textbooks for very high operation frequency. If $R=\omega L_{i}$ can be assumed, the internal inductance $L_{i}$ can be trivially obtained from the calculated value of $R$. However, this assumption is what is considered wrong or inaccurate in the above paper for the specific case of rectangular cross-sectional conductors. On the other hand, the authors of the above paper raise a question about the foundations of the well-known Wheeler's rule when applied to rectangular conductors. Apparently Wheeler's rule relies on the high-frequency resistance and internal inductive reactance being equal. However, although this premise seems to be violated in the case of rectangular conductors, Wheeler's rule yields reasonably accurate results in this case. We would like to share with the authors of the above paper and other interested colleagues some considerations concerning the above-mentioned conclusions and paradox.

From the reading of section III in the above paper, it seems that the authors attribute the difference between the real $(R)$ and imaginary $\left(\omega L_{i}\right)$ parts of the internal impedance to the existence of right-angle corners in the rectangular cross-sectional geometries. These corners would be responsible for the nonconstant distribution of the currents along the periphery of the conductors, and this fact makes it a different internal resistance and reactance. However, in our opinion, there is a problem hidden in this argument. In order to illustrate the nature of the problem, let us compare some numerically computed results reported in the above paper with results analytically obtained for two simpler geometry wires having the same dc resistance $\left(R_{\mathrm{dc}}\right)$. The cross sections of the structures under comparison are shown in Fig. 1, where conductivity and dimensions are given. Note that the surface of the cross sections and the conductivity are the same for all the three wires, thus, they have all the same value of $R_{\mathrm{dc}}$. Fig. $1(\mathrm{~b})$ is an idealized version of the square wire considered in the above paper [see Fig. 1(a)], where corner effects are suppressed by imposing magnetic boundary walls at two parallel sides. Closed-form expressions for the internal impedance of this structure are readily obtained through elementary calculations. These expressions are valid for any frequency, including

Manuscript received November 9, 2000.

The authors are with the Microwaves Group, Department of Electronics and Electromagnetism, University of Seville, 41012 Seville, Spain (e-mail: medina@cica.es; marques@cica.es).

Publisher Item Identifier S 0018-9480(01)06143-9.

${ }^{1} \mathrm{G}$. Antonini, A. Orlandi, and C. R. Paul, IEEE Trans. Microwave Theory Tech., vol. 47, no. 7, pp. 979-985, July 1999.

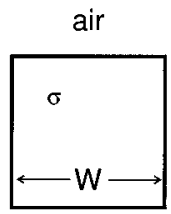

air

(a)

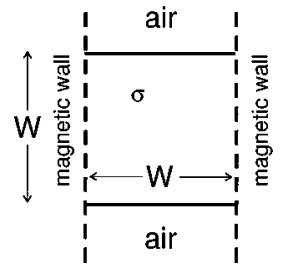

(b) air

(c)

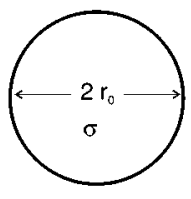

Fig. 1. Cross section of the wires whose internal impedances are compared in Fig. 2. (a) An example structure analyzed in Fig. 6 of the above paper. (b) The same structure with magnetic walls eliminating corner effects. (c) Round wire having the same dc resistance as the previous wires. Dimensions: $W=$ $4.62 \mathrm{~mm}, r_{0}=W / \sqrt{\pi}$. Conductivity: $\sigma=5.72 \times 10^{7}(\Omega \cdot \mathrm{m})^{-1}$.

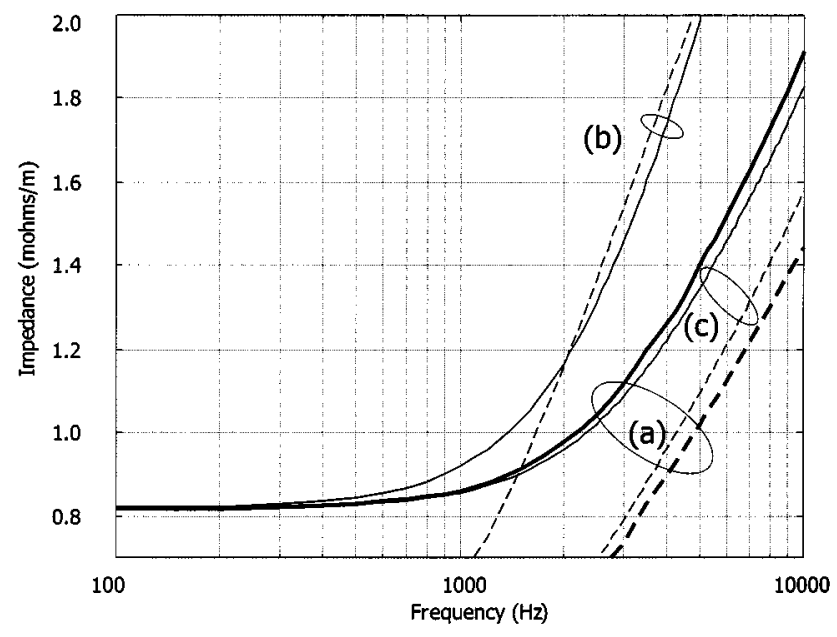

Fig. 2. Internal resistances (solid lines) and reactances (dashed lines) of the wires in Fig. 1. Thick lines correspond to data appearing in Fig. 6 in the above paper for the structure in Fig. 1(a).

negligible to strong skin-effect operation. Fig. 1(c) is a cylindrical circular wire whose internal impedance is known in closed form in terms of Bessel functions for any frequency (see, e.g., [1, pp. 180-186]). We have reproduced in Fig. 2 the numerically computed data reported in Fig. 6 in the above paper [internal resistance and reactance of the structure in Fig. 1(a)] and analytical exact data for the structures in Fig. 1(b) and (c). We can see that the real and imaginary parts of the internal impedance are very close in the case of Fig. 1(b). There is a small difference because, for the range of frequencies included in this figure, the skin depth is not yet negligible in comparison with $W$. However, it is true that if frequency is increased, $R$ becomes identical to $\omega L_{i}$ in this case. However, the internal impedance of the circular cross-sectional wire [see Fig. 1(c)] presents a behavior very similar to the one of the square cross-sectional wire, even though the round wire obviously has no corners. In our opinion, for the structure shown in Fig. 1(b), we find $R=\omega L_{i}$ (when skin effect is developed) because the electromagnetic field (EMF) inside the conductor is exactly a uniform plane wave, as assumed in the derivation of the high-frequency internal impedance formulas, and not only because the EMF is constant along the periphery of the conductor. In fact, the EMF is uniform around the circular wire surface, but $R \neq \omega L_{i}$ because a cylindrical wave instead of a plane wave exists inside the conductor. The cylindrical solution approaches 
the plane wave limit only if $\delta \ll r_{0}$ ( $\delta$ is the skin depth). This result can be generalized saying that, in order to have $R \approx \omega L_{i}$, the curvature radius must be large in comparison with the skin depth. In the case of rectangular cross-sectional conductors, this condition is not fulfilled in the vicinity of the corners. Thus, even though each side is a planar surface, the EMF is strongly nonuniform along the conductor surface (as it is stated in the above paper), thus providing a place to an inner EMF that is not the one assumed in the derivation of the conventional strong skin-effect formulas. There is still a noteworthy point to be emphasized in connection with this question. From inspection of Fig. 2 and from analytical calculations, we can verify that, if the skin effect is strong, $R \approx \omega L_{i}$ in the sense $\left(R-\omega L_{i}\right) \rightarrow 0$ when $\omega \rightarrow \infty$, only in the case of the structure in Fig. 1(b). In the case of the round wire [see the structure in Fig. 1(c)], it can be readily demonstrated that $R-\omega L_{i} \rightarrow 0.25 R_{\mathrm{dc}}$ when $\omega \rightarrow \infty$ (this difference between $R$ and $\omega L_{i}$ can be appreciated, e.g., in [1, Fig. 4.5(a) and (b)]). This difference is negligible for very high frequencies because, in such case, $R \approx \omega L_{i} \gg R_{\mathrm{dc}}$. Therefore, we can always say that $R /\left(\omega L_{i}\right) \rightarrow 1$ when $\omega \rightarrow \infty$, and it is in this sense that Wheeler formulas are correct. However, the above-mentioned difference becomes important when the cross-sectional dimensions are just a few times the skin depth, as happens in Fig. 2. This is the difference that is observed in the curves shown in the above paper. As can be seen, this difference cannot be considered a specific feature of rectangular cross-sectional conductors or exclusively related with the presence of corners. Note that, if we are correct, all this would provide an answer to the open question formulated in the last sentence of the above paper, namely, "The reason why Wheeler's rule seems to give reasonably accurate values of resistance when skin effect is well developed even though the basic premise is violated is not understood." The problem is in the concept "well developed skin effect." The authors of the above paper seem to consider that the skin effect is well developed if the transverse dimensions of the wire are a few times the skin depth. However, from the study of the circular wire, it is obvious that the resistance coincide with the "high frequency" (Wheeler) formulas when skin depth is meaningfully small in comparison with the curvature radius of the conductor contour. If this condition is not fulfilled, Wheeler's expressions would be just approximated, independently of the existence of corners. However, it is true that the existence of corners makes the difference between $R$ and $\omega L_{i}$ larger than if corners were not present, but this is a rather obvious result.

There is still another point deserving attention in connection with conductors having right angles such as those involved in this paper. Let us consider the square conductor of the example in the previous paragraph as one of the conductors of a two-wire transmission line. The total per unit length (p.u.l.) inductance of the transmission line, if strong skin-effect operation is assumed, can be usually split into two contributions: the external inductance, associated to the magnetic field existing in the dielectric medium around the wire, and the internal inductance, associated to the magnetic field penetrating the conductor. The external inductance is considered to be identical to the inductance of the transmission line when the conductors are considered perfect. This assumption is justified at any frequency (including weak skin-effect operation) for structures having a high degree of symmetry. For instance, the external inductance of a conventional coaxial transmission line is the same under weak or strong skin-effect conditions: the external magnetic field does not depend on the particular distribution of the current inside the inner conductor, whereas cylindrical symmetry is respected. For more general situations, the assumption of an external magnetic field identical for the line made of perfect and nonperfect conductors is valid if the skin depth is very small in comparison with local curvature radius of the contour curve describing the geometry of the conductor. It is clear than for rectangular cross-sectional wires, the external magnetic field is not identical to the field for the structure with perfect conductors, at least in the neighborhood of the corners. Therefore, we cannot rigorously say that the total inductance is the summation of the internal inductance and the inductance of the same structure made of perfect conductors. A more general interpretation of the model would be based on the consideration of an incremental inductance associated to the nonperfect nature of the conductors rather than an internal inductance. This incremental inductance would be the difference between the total inductance of the lossy line and the total inductance of the lossless line. This parameter would not coincide with the internal inductance computed from the internal magnetic energy stored inside the conductors, such as it is carried out in the above paper. The external inductance could be calculated from the computed values of the current density and magnetic potential vector inside the lossy conductors since the expression of the magnetic energy in terms of such quantities includes the true external magnetic energy. Now, by substracting the inductance of the lossless transmission line, we have the incremental inductance, which should be used to model the inductive effect due to the lossy nature of the conductor instead of the internal inductance. Obviously, for very strong skin-effect operation or for conductors having smooth contours, the distinction between incremental and internal inductance has no meaning. It would be interesting to explore which of those inductances (incremental or internal) yields a reactance closer to the ac resistance in the case of rectangular conductors or conductors having corners.

\section{REFERENCES}

[1] S. Ramo, J. R. Whinnery, and T. van Duzer, Fields and Waves in Communication Electronics, 3rd ed. New York: Wiley, 1994.

\section{Authors' Reply}

Giulio Antonini, Antonio Orlandi, and Clayton R. Paul

The authors of the above paper, ${ }^{1}$ intended to demonstrate the following point about conductors that have rectangular cross sections. The per-unit-length resistance $r$ and per-unit-length internal inductive reactance $\omega l_{i}$ are considerably different at high frequencies where skin effect is well developed, which is in contrast to conductors of circular-cylindrical cross section (wires). There is the question of what is meant by skin effect being "well developed." First, consider the case of a wire of radius $r_{w}$. We compare that radius to a skin depth $\delta=1 / \sqrt{\pi f \mu \sigma}$, where $f$ is the frequency of excitation,

Manuscript received February 1, 2001.

G. Antonini is with the Department of Electrical Engineering, University of Roma "La Sapienza," Rome 00184, Italy.

A. Orlandi is with the Department of Electrical Engineering, University of L'Aquila, 67040 Poggio di Roio, L'Aquila, Italy.

C. R. Paul is with the School of Engineering, Mercer University, Macon, GA 31207 USA.

Publisher Item Identifier S 0018-9480(01)06144-0.

${ }^{1} \mathrm{G}$. Antonini, A. Orlandi, and C. R. Paul, IEEE Trans. Microwave Theory and Tech., pp. 979-985, vol. 47, no. 7, July 1999. 